\title{
Transport Stream Playout System for MPEG-TS using Program Clock Reference
}

\author{
Anali D Shah \\ Ganpat University, Kherva,
}

\author{
Sudhir Agrawal \\ Space Applications Centre, \\ ISRO, Ahmedabad
}

\author{
Kapil Sharma \\ Space Applications Centre, \\ ISRO, Ahmedabad
}

\begin{abstract}
This paper presents the working and implementation of streaming rate control mechanism in real time video streaming over IP for Digital Video Broadcasting using PCR (Program Clock Reference). DVB (Digital Video Broadcasting) supports MPEG-TS mode of transmission such that videos are encoded in transport streams. Moving video images must be delivered in real time and with a consistent rate of presentation in order to preserve the illusion of motion. The PCR is a time reference that is sequentially transmitted with each program of a transport stream. PCR refers to the timing information for proper synchronization of audio and video which simultaneously control the rate of the packet transmitted. For sequential streaming of multiple MPEG-TS parameters in PAT (Program Association Table)are required to be same for all programs being streamed. PAT table is generated in transport stream packet with specific PID values.
\end{abstract}

\section{Keywords}

Digital Video Broadcasting-DVB, Transport Stream-TS, Program Clock Reference-PCR, Program Association TablePAT

\section{INTRODUCTION}

With the ongoing demand of Direct to Home (DTH) services through the DVB over Satellite, enlightens the need of the packet switched network integration in the services [1]. The Audio, video and data are carried in the TS packet according to the ISO-13818 standards. The Transport Stream is used for communication in error prone environment and having packet size of 188 bytes.

This paper presents the software based Transport Stream playout system along with the implementation of sequential streaming mechanism for multiple TS files. Transport stream playout system reads a transport stream from a file and broadcast it over a high-speed Ethernet interface in specific packet formats eg. UDP, ICMP, TS etc at the appropriate or required speed [2]. TS files are streamed via Ethernet using a simple Windows based GUI application. Streaming is such a way that play-out of audio and video fragments into number of packets and received in the form of video frames. Digital television distribution systems require for processing not only complete transport streams offered by the program suppliers, but also several kinds of additional information like PSI (Program Specific Information) tables and clock reference.
The player gets its source information from the local storage and transports to the receiver with controlling the packets which is in TS format. In this paper streaming rate control mechanism is given using PCR. PCR is a clock reference which is generated in TS packets in specific period of time. TS streaming can conceptually be thought to consist of the following steps [3]:

1. Playing out the transport stream at the right flow

2. Getting the transport stream into a format that receiver can understand

With controlled streaming rate, one TS file is transmitted from sender to the receiver in TS format. After streaming of single file, same mechanism is applied for sequential streaming of multiple TS file. However parameters in PAT need to be kept same in TS packet.

The flow of playout system is as follow:

1) Partition the compressed video into packets using PCR

2) Start delivery of these packets

3) Playback at the receiver while the video is still being delivered

4) After completion of one stream start from step one, however transmission parameters for PAT remains same.

TS streaming enables simultaneous delivery and playback of the video. In TS streaming there usually is a short delay (order of 2-5 milliseconds) between the start of delivery and the beginning of playback at the client. TS streaming provides a number of benefits including low delay before viewing starts and low storage requirements since only a small portion of the video is stored at the client at any point.

\section{MPEG-TS AND PCR CHARACTERISTICS}

MPEG transport stream is a standard format for transmission of audio, video, and program \& System Information Protocol (PSIP) data. It is used in broadcast systems such as DVB, ATSC and IPTV [2].

$\operatorname{pcrBase}(I)=\left[\left(\begin{array}{c}\text { systemClockFrequency } \\ * t(I)) \text { DIV 300]MOD } 2^{33}\end{array}\right.\right.$ 


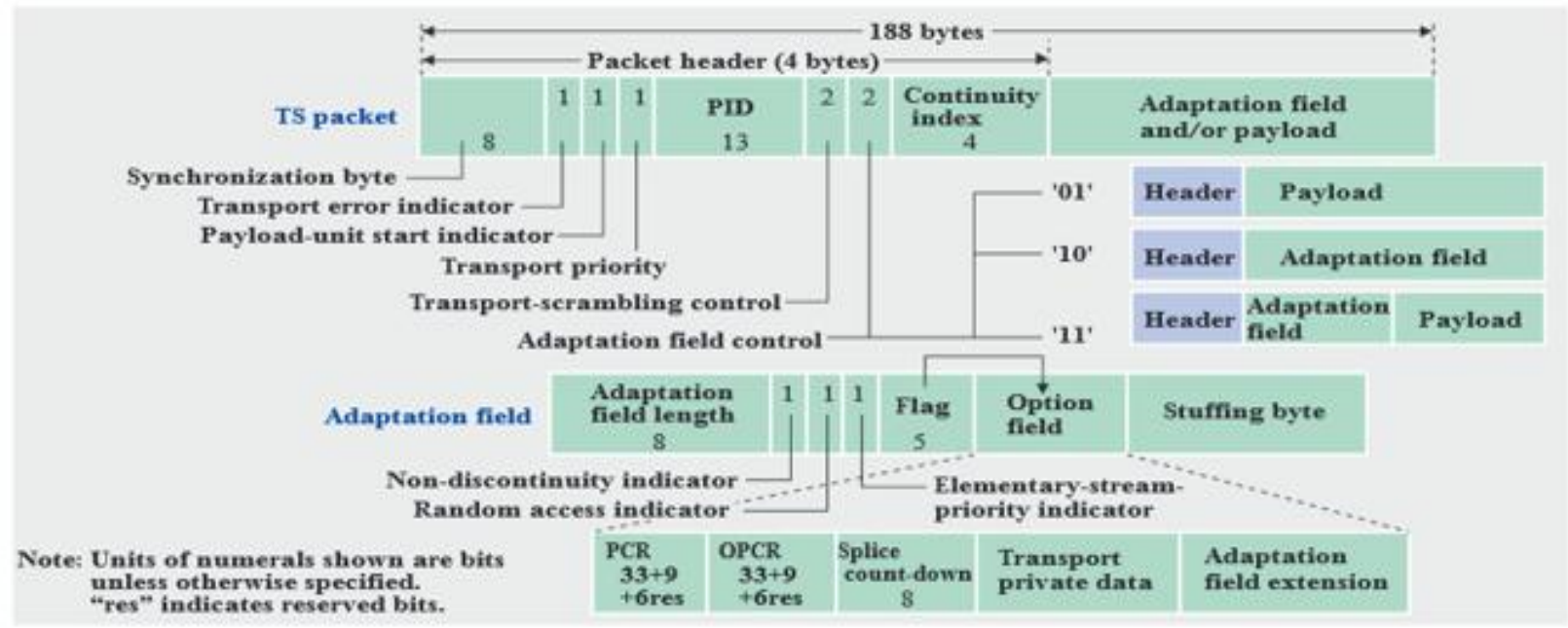

Fig 1: Transport Stream Packet Format [4]

\section{pcrExtension $(I)=[($ systemClockFrequency * t(I))DIV 1] MOD 300}

MPEG-2 transport stream is suitable for transmissions when there is potential corruption or loss of packets. Transport stream consists of 188-byte fixed-length packets. The Transport Stream rate is defined by the values and locations of Program Clock Reference (PCR) fields, which in general are separate PCR fields for each program. PCR value is transmitted in an optional field of the extendable header (adaptation field) in the transport stream packet as shown in Figure1.The PCR is required in order to synchronize the transmitter and receiver by transmitting the uniform $27 \mathrm{MHz}$ System Time Clock (STC)[5].

The PCR values of a program are only transmitted in transport stream packets having a PID, which is specified in the Program Map Table (PMT). This is usually the video elementary stream of the program. Not every TS packet containing this specific PID necessarily includes a PCR Value [6]. It is sufficient to insert a value into a TS packet every 100 $\mathrm{ms}$ (according to DVB/MPEG). The PCR value is 42 bits long and incremented with a frequency of $27 \mathrm{MHz}$. PCR is made up of a 33-bit field of Program Clock Reference Base and a 9bit field of Program Clock Reference Extension. The final PCR value is calculated using formula [6]:

$\operatorname{PCR}(I)=\operatorname{pcrBase}(I) * 30+\operatorname{pcrExtension}(I)$

This PCR value is helpful in streaming rate control mechanism for TS playout system.

\section{PROPOSED APPROACH}

\subsection{Streaming Rate Control Mechanism Using PCR}

The pre stored video file is to be streamed to receiver end. The video file is first converted to TS format and streamed. Transport stream has a fixed size packet format of 188 Bytes. For conversion of different format of video file to TS format, software like VLC player, TS converter etc may be used. TS packet contains 4 Byte header and 184 Byte payload or adaptation field. A packet can have both fields.

The TS header is extracted and bit checking is done to find the presence of PCR (Program Clock Reference) in adaptation part of Transport Stream. One Counter is used to determine the number of packets between two PCR. Absence of PCR results in increment of non-PCR packet count.

As discussed, 42 bits long PCR field is extracted from file, comprising of PCR Base ( 33 bits) and PCR Extension (9 bits). PCR Value is calculated using these base and extension bits. Formula to calculate its value (in millisecond):

PCRvalue $=\left(\frac{\text { pcrBase } * 300+\text { pcrExtension }}{27 M H z}\right) * 1000$

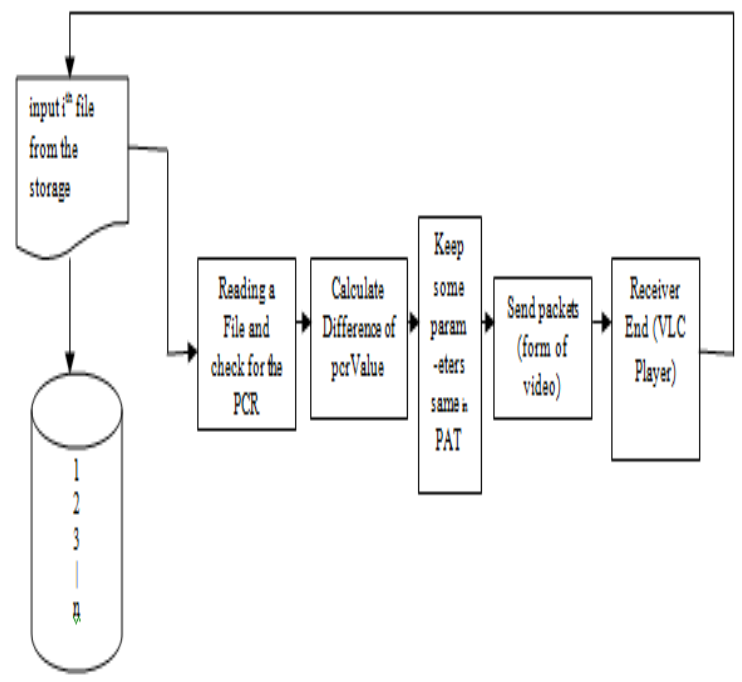

Fig 2: Playout System

Theoretically, PCR is generated every $100 \mathrm{~ms}$ at $27 \mathrm{MHz}$ frequency. However it is observed that PCR stamp or tag it is generated every 70 to $80 \mathrm{~ms}$.

For every PCR, the numbers of packets between two consecutive PCR are calculated on the basis of time difference. The number of packets between two PCR stamps varies each time and cannot be null.

$$
\operatorname{pcrDifference}=\operatorname{pcrValue}\left(I^{\prime \prime}\right)-\operatorname{pcrValue}\left(I^{\prime}\right)
$$


The numbers of packets are controlled using this PCR value in milliseconds. These packets are sent in the PCR time difference calculated above to control the rate of flow of packets leading to no loss of packets or any malformed packets. The TS file is regenerated and stream to receiver with streaming rate control mechanism through PCR technique and played in the VLC Player.

\subsection{Sequential Streaming of Multiple MPEG-TS}

With controlled streaming rate, transmission of MPEG-TS to the receiver is achieved. At the receiver VLC player is used to play the TS video file and only single streaming is achieved. Then another task is sequential streaming of multiple MPEGTS like one by one transmission of MPEG-TS to the receiver end. For this purpose there is need to keep few parameters of all TS same. Then VLC can easily decode it and play same as streaming rate control mechanism. TS contain some Program Specific Information or PSI tables. There are two tables in TS which are very important for such kind of transmission and these are Program Association Table and Program Map Table. All this information in TS files come in TS packets with some specific PID values. There are two parameters i.e. Transport Stream ID and Version Number in PAT table that should be same for all TS. PMT table contains audio-video information about TS. New CRC values are re calculated according to changes. Otherwise dilemma can occur at the receiver side like packet loss or malformed packet. After applying this, receiver can easily decode it and sequential streaming of multiple MPEG-TS transmission is achieved.

\section{IMPLEMENTATION STRATEGY AND RESULTS}

The converted or encoded TS file is read and numbers of packets between two PCR values are calculated and send in this duration. The numbers of packets between two PCR are not fixed. Figure 3 shows the packets between two PCR of different length.

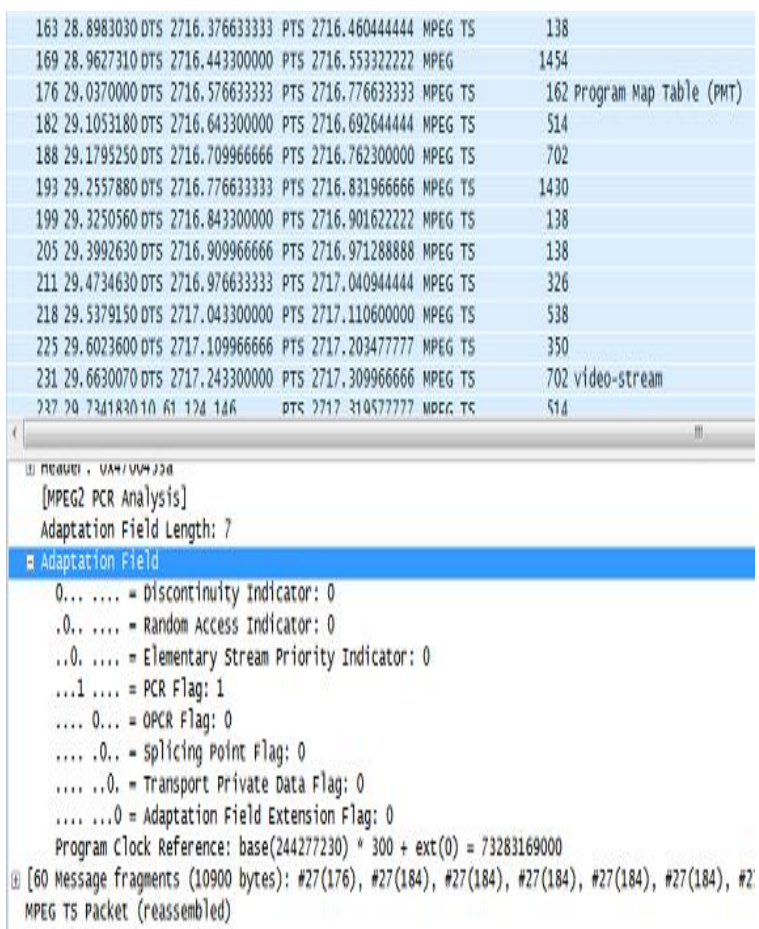

Streaming of TS file with PCR strategy can play the transport stream to the receiver side. We can play this transport stream at receiver using VLC player by setting in open network stream. Figure 4 shows that VLC plays TS stream with very fine quality and less distortion.

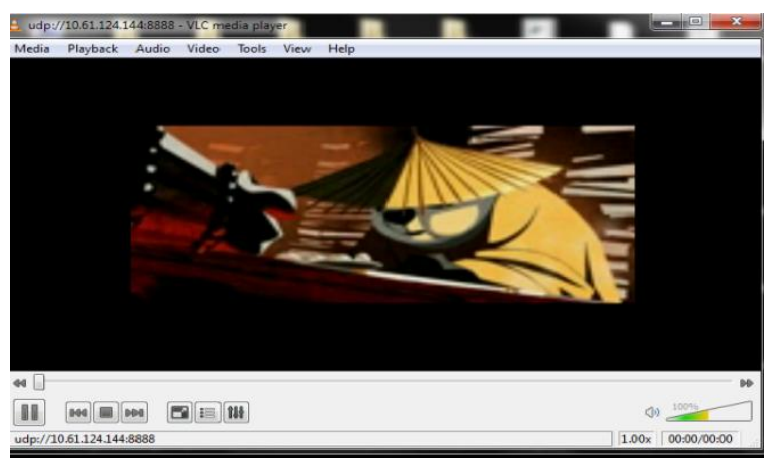

Fig 4: Streaming at receiver point (VLC Player)

TS reader checks the following parameters of TS packets like PAT, PMT, CRC, continuity errors etc. in case of presence of any errors in TS file, TS reader cannot decode video and other parameters of TS and displays number of continuity and CRC errors. Otherwise it displays audio and video stream with its PID values and bit rate of the transport stream. Figure 5 shows captured data of TS file with no errors.
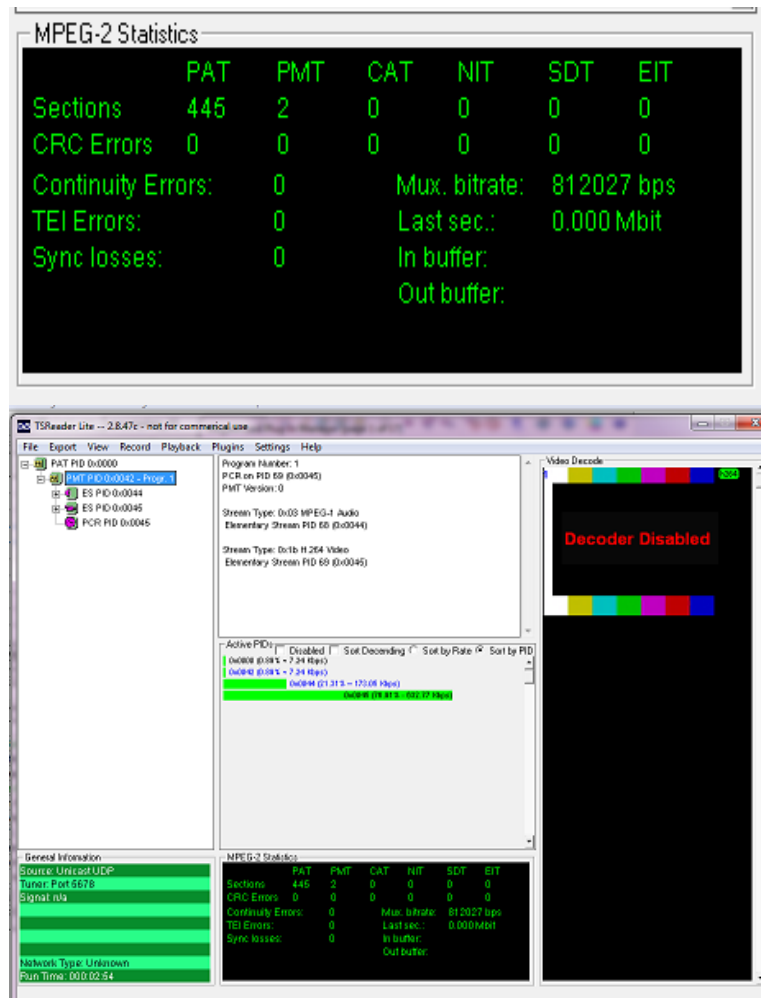

Fig 5: TS Streaming (TS Reader)

For sequential streaming, TS file parameters are changed. Transport stream ID and version number are kept same in Program Association Table in TS file. This can be applied for all TS file. Hence, VLC can easily play TS file one by one. Figure 6-9 shows sequential streaming for 4 TS file and it can work for several numbers of streams.

Fig 3: Packets between two PCR (Wireshark) 


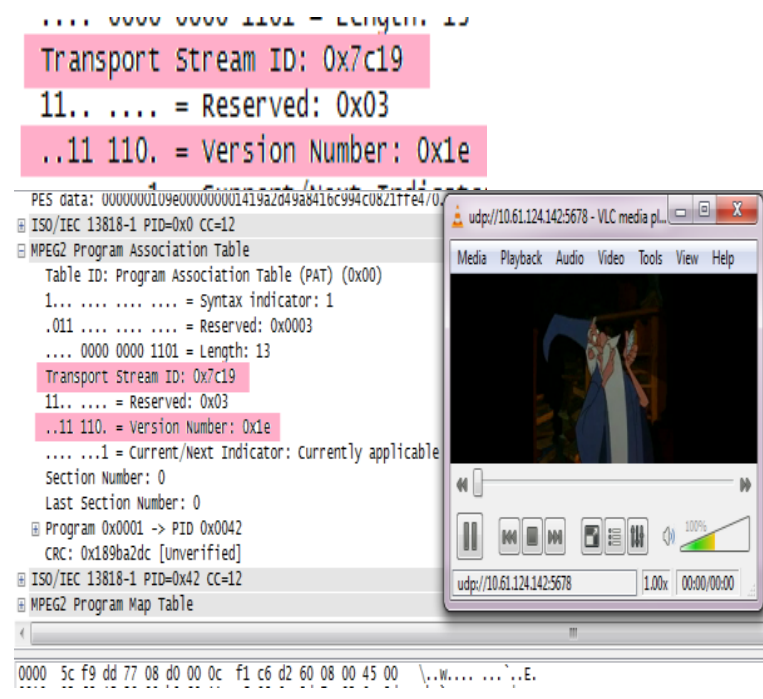

Fig 6: Sequential streaming of multiple MPEG-TS (Wireshark, VLC Player) -first file in the list

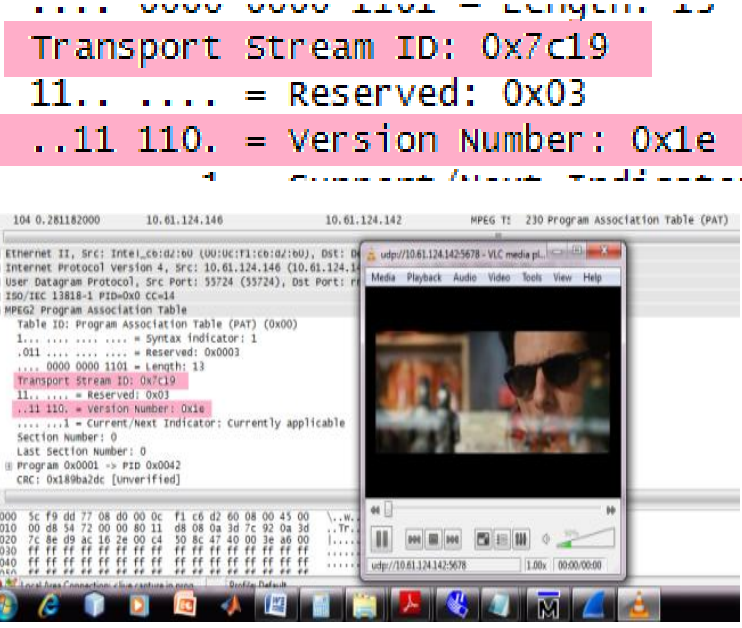

Fig 7: Sequential streaming of multiple MPEG-TS (Wireshark, VLC Player) -second file in the list

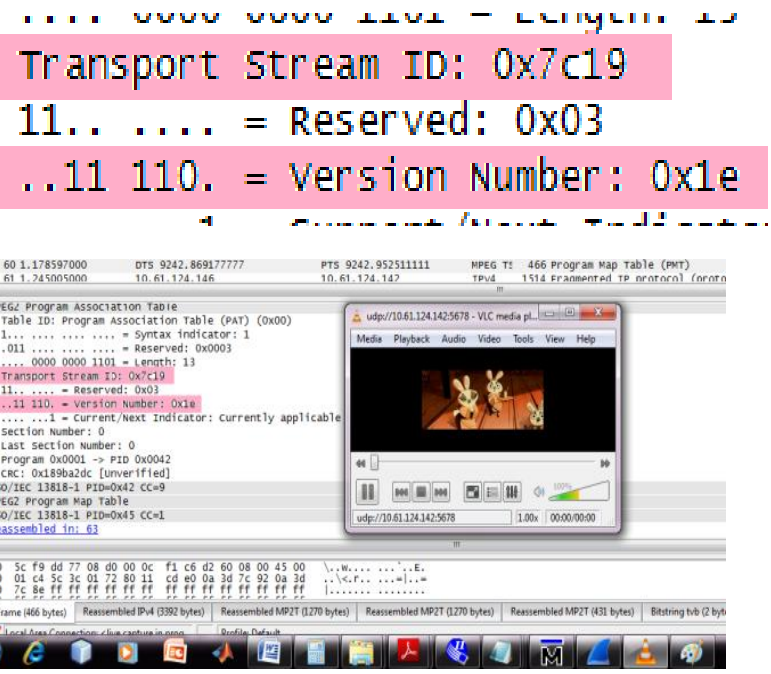

Fig 8: Sequential streaming of multiple MPEG-TS (Wireshark, VLC Player) -third file in the list

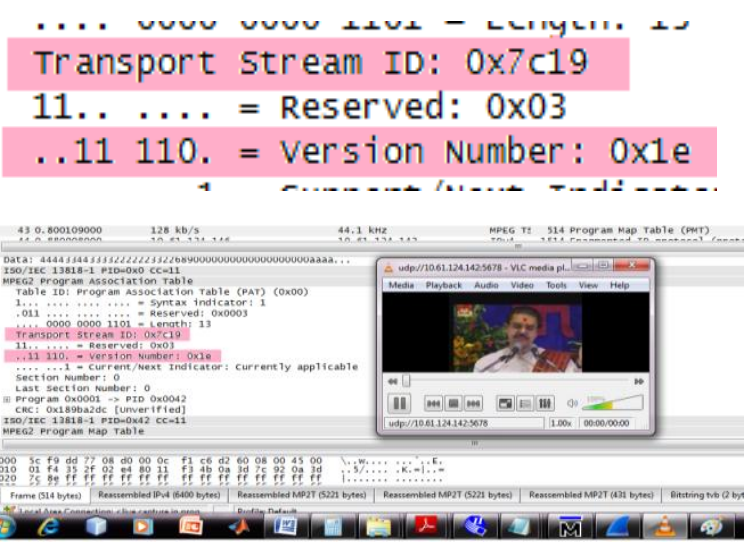

Fig 9: Sequential streaming of multiple MPEG-TS (Wireshark, VLC Player) -fourth file in the list

\section{CONCLUSION}

The intended goal of packet control and sequential streaming of multiple MPEG-TS is achieved. The playout system works perfectly. TS streaming can be done and video plays in VLC player successfully one by one. Packets captured in wireshark between two PCR and audio video stream and other TS parameters are also be shown in TS reader but still there are some minor delay in presenting the first stream. Further work on the time by time playout system and streaming can be done on multiple channels.

\section{ACKNOWLEDGMENT}

Authors are thankful to Shri Kaushik Parikh, Dy Director, SNAA/SAC/ISRO and Shri Virender Kumar, GH, SSTG/SNAA/ISRO for their continuous guidance, encouragement and support. The authors are indebted to Head \& Prof Dr.Kiran Amin, and Prof Rakesh Vanzara, Ganpat University, Kherva for their guidance. The authors sincerely appreciate the critical evaluation and constructive suggestions provided by the reviewers.

\section{REFERENCES}

[1] ETSI TR 101 290: "Digital Video Broadcasting (DVB) Measurement guidelines for DVB systems"

[2] ISO-13818/1-“Generic Coding of Moving Pictures"

[3] http://www.interactivetvweb.org/tutorials/getting_started /application_development/generating_a_transport_stream .html

[4] Corona Publishing - "Lecure pdf on Overview of MPEG2 Systems"

[5] Alexandru - "A new method for calculating PCR in packet based transmission network"

[6] Thesis - "Comprehensive PCR Analysis Using the $\& S \circledast D V M$ Family from Rohde \& Schwarz R\&S®DVM50/DVM100/DVM20/DVM400” 